\title{
Mapping Property of Bilateration and Its Application to Human-Following Robot
}

\author{
Heungju AHN ${ }^{\mathrm{a}}$, Van Chien DANG ${ }^{\mathrm{b}}$, Hyeon Cheol SEO ${ }^{\mathrm{b}}$, and Sang C. LEE ${ }^{\mathrm{b}, 1}$ \\ ${ }^{a}$ College of Transdisciplinary, DGIST, Daegu, 42988, Rep. of Korea \\ ${ }^{\mathrm{b}}$ IO Lab, Division of Intelligent Robot, Convergence Research Institute, DGIST, Daegu, \\ 42988, Rep. of Korea
}

\begin{abstract}
Objective of this paper is twofold. The first one is to study the mapping property and unified form of the component equations of the unknown node in bilateration, and the second one is to introduce the concept model for humanfollowing robot based on bilateration. Bilateration needs only two known nodes and two distances' data. Because of the simple sensor arrangement in bilateration, it needs less computation and uses less number of unavoidable erroneous distances compared to the trilateration.
\end{abstract}

Keywords. trilateration, bilateration, human-following, mapping property, hyperbola

\section{Introduction}

The Internet of Things (IoT) is a system that connects different 'things' to provide ubiquitous connectivity and enhanced services [1] and has an extensive set of applications in view of consumer, commercial, industrial, and infrastructure spaces [2]. Up to now, the technology of IoT has been evolved focused on sensing, collection of information, and communication [3]. In this situation, if the position information is added to each IoT, then IoT can provide much wider range of services [1].

The purpose of this paper is twofold; the first objective is to reveal several mathematical properties of the bilateration method; the second one is to propose a bilateration method based on moving frame for the concept model of the human-following robot.

The trilateration in two dimensional geometry, i.e., in $\mathbb{R}^{2}$ is most well-known method to determine the unknown node from three different known nodes ${ }^{2}$ [4]. Bilateration in $\mathbb{R}^{2}$ is a method to determine the coordinates of a movable or stationary point (which will be called unknown node) using two measured distances between two distinct known node and unknown node. However, as shown in Figure 1a, two measured distances in bilateration do not give the exact coordinates of the unknown node, but fortunately, there

\footnotetext{
${ }^{1}$ Corresponding Author (E-mail:sclee@ dgist.ac.kr)

This work was partly supported by DGIST R\&D program of the Ministry of Science, ICT, and Future Planning of KOREA (20-IT-03) and Korea Institute of Energy Technology Evaluation and Planning (KETEP) grant funded by the Korea government (MOTIE) (2019-3010032490, Development of 25kW modular fuel cell power conversion system)

${ }^{2}$ Different terminology is 'stations' or 'anchor'
} 
are finitely many (two except the points on the $x$-axis) candidates. To choose the desired one from two candidates, we suggest the bilateration, which is acting on the moving robot (see Figure 3b).

So far, Heron-bilateration method [5,6,7] has been proposed by many researchers. Unlike our bilateration method mounted on the mobile robot, they all ask somehow $a$ priori knowledge about the unknown node.

The bilateration has more advantages compared to trilateration in the following sense: to reduce the number of required known nodes, to require less computation and time to response, and also to provide better position accuracy, since it uses less number of inaccurate distance in computation $[5,6,7]$.

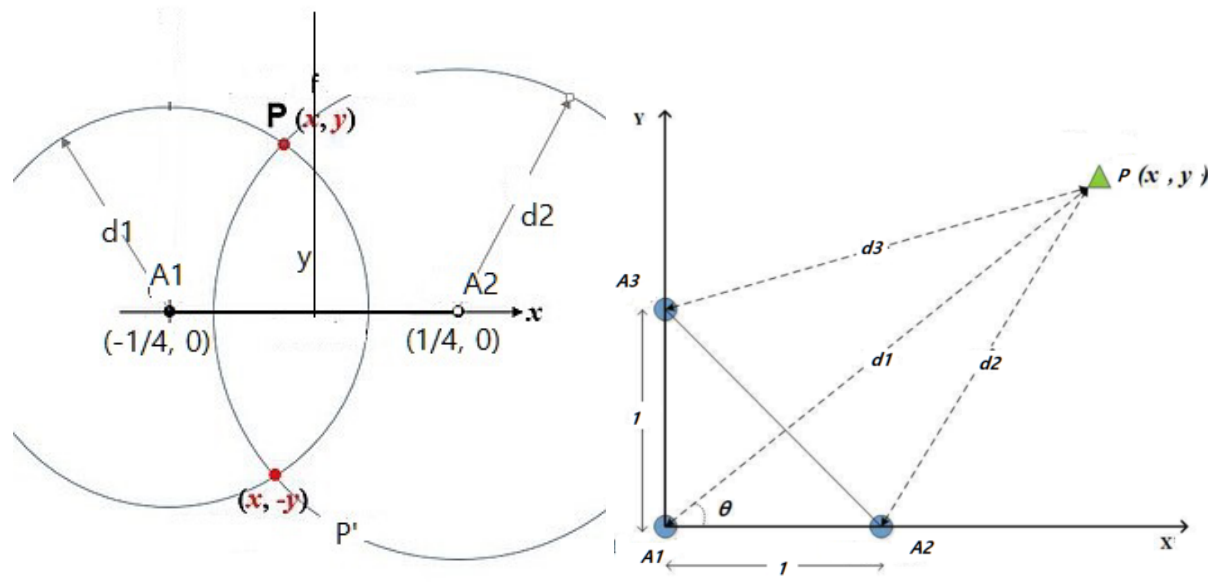

(a) Bilateration: $P$ is unknown node and $A_{1}, A_{2}(\mathrm{~b})$ Trilateration: $P$ is unknown node and are known nodes. $A_{1} \cdot A_{2} \cdot A_{3}$ are known nodes.

Figure 1. Bilateration and Trilateration

\section{Properties of Bilateration}

In this section we prove that the bilateration is the most efficient method among the multilateration in $\mathbb{R}^{2}$ if we have a method to choose the exact coordinates of the unknown node among two candidates. Actually, trilateration method is the following: bilateration gives information to narrow the possible positions down to two candiates, and then another distance measurement from a known node that is different from two known nodes in bilateration determines the exact position.

As we shall see it in $\S 3$, there is another way of choosing the exact position by moving the one of the two known nodes. In this case, bilateration has more advantage compared to the trilateration, since we have only two measurement errors and the process for the additional information conains no more numerical error.

Also, as a main contribution of this paper, we study the mapping properties of the bilateration and show the $x$ - and $y$-components of the coordinates of the unknown node obtained from the bilateration method have similar forms as trilateration under the suitable change of variables. 


\subsection{Generalized Geometry Problem}

Let $\mathbb{R}^{2}$ be two dimensional Euclidean space equipped with the Cartesian coordinate system. Let $A_{1}, A_{2}, \ldots, A_{n} \in \mathbb{R}^{2}$ be $n$-distinct nodes in the plane whose coordinates are known and $P \in \mathbb{R}^{2}$, an unknown node. Further, assume that non-negative scalar-valued numbers $d_{1}, d_{2}, \ldots, d_{n}$ are given, where each $d_{k}(k=1,2, \ldots, n)$ is obtained from the information ${ }^{3}$ between $A_{k}$ and $P$. The generalized geometry problem is to determine the coordinates of the unknown node $P$ from known nodes $A_{1}, A_{2}, \ldots, A_{n}$ using measurement data $d_{1}, d_{2}, \ldots, d_{n}$.

Since the space filling curve can not be one-to-one, one known node $A=A_{1}$ and one measured datum $d=d_{1}$ can not uniquely determine every position in $\mathbb{R}^{2}$. It is possible to approximate the position in $\mathbb{R}^{2}$ instead of filling it. This kind of localization corresponds to the empirical method to match the information from the unknown location with a sufficiently large data of known locations.

\subsection{Bilateration}

In this section, we explain the bilateration method using the distance data. For the simplicity of notations, let $A_{1}=(-1 / 4,0), A_{2}=(1 / 4,0)$ be two known nodes, and $P=(x, y)$, unknown node to be determined using $d_{1}$ and $d_{2}$ (recall $d_{1}$ is the distance between $A_{1}$ and $P$, and $d_{1}$ is the distance between $A_{2}$ and $P$ ).

Simple observation (see Figure 1a) gives

$$
\begin{aligned}
& (x+1 / 4)^{2}+y^{2}=d_{1}^{2}, \\
& (x-1 / 4)^{2}+y^{2}=d_{2}^{2} .
\end{aligned}
$$

Subtracting (2) from (1), we have

$$
x=d_{1}^{2}-d_{2}^{2}
$$

and plugging (3) into (1) and arranging the terms, we obtain

$$
y^{2}=d_{1}^{2}-\left(d_{1}^{2}-d_{2}^{2}+1 / 4\right)^{2}
$$

Theorem 2.1. The function $\left(d_{1}, d_{2}\right) \mapsto\left(x\left(d_{1}, d_{2}\right), y\left(d_{1}, d_{2}\right)\right.$, which will be called a bilateration mapping, defined by (3) and (4)

$$
\begin{aligned}
& x\left(d_{1}, d_{2}\right)=d_{1}^{2}-d_{2}^{2} \\
& y\left(d_{1}, d_{2}\right)=\sqrt{d_{1}^{2}-\left(d_{1}^{2}-d_{2}^{2}+1 / 4\right)^{2}}
\end{aligned}
$$

is a bijective mapping from the domain $S=\left\{(x, y) \in \mathbb{R}^{2}: d_{1}+d_{2} \geq 1 / 2, d_{2} \leq d_{1}+\right.$ $\left.1 / 2, d_{2} \geq d_{1}-1 / 2\right\}$ to the upper half plane set $H=\left\{(x, y) \in \mathbb{R}^{2}: x \in \mathbb{R}, y \geq 0\right\}$. Also, the function $x, y$ are differentiable in the interior of $S$, and preserves the boundary.

\footnotetext{
${ }^{3}$ The most typical information is the Euclidean measured distance $d_{k}$ between $A_{k}$ and $P$ using sensors.
} 
Proof. Since three points $A_{1}, A_{2}$, and $P$ form a triangle, and the length of the line segment $\overline{A_{1} A_{2}}$ is exactly $(1 / 2)$, we have the first restriction equation

$$
d_{1}+d_{2} \geq 1 / 2
$$

Next, note that $y^{2}=d_{1}^{2}-\left(d_{1}^{2}-d_{2}^{2}+1 / 4\right)^{2} \geq 0$. Solving this inequality, we have two more equations

$$
\begin{aligned}
& 0 \leq d_{1}-\left(d_{1}^{2}-d_{2}^{2}+1 / 4\right)=d_{2}^{2}-\left(d_{1}-1 / 2\right)^{2}, \quad \text { and } \\
& 0 \leq d_{1}+\left(d_{1}^{2}-d_{2}^{2}+1 / 4\right)=\left(d_{1}+1 / 2\right)^{2}-d_{2}^{2} .
\end{aligned}
$$

Solving the above inequalities simultaneously and noticing that $d_{1}, d_{2}$ are non-negative real numbers, we obtain the restrictions

$$
d_{1}-1 / 2 \leq d_{2} \leq d_{1}+1 / 2
$$

that define the domain $S$.

Finally, we have to check how the bilateration mapping maps the boundary of $S$ onto that of $H$. The boundary of $S$ is composed of three parts: $d_{1}+d_{2}=1 / 2(0 \leq$ $\left.d_{1}, d_{2} \leq 1 / 2\right) ; d_{2}=d_{1}-1 / 2\left(1 / 2 \leq d_{1}\right)$, and $d_{2}=d_{1}+1 / 2\left(0 \leq d_{1}\right)$. From the equality $d_{2}=d_{1}-1 / 2$, the straightforward calculation shows

$$
\begin{aligned}
x\left(d_{1}, d_{2}\right) & =d_{1}^{2}-d_{2}^{2}=d_{1}-1 / 4\left(0 \leq d_{1} \leq 1 / 2\right) \\
y\left(d_{1}, d_{2}\right)^{2} & =d_{1}^{2}-\left(d_{1}^{2}-d_{2}^{2}+1 / 4\right)^{2}=d_{1}^{2}-\left(d_{1}^{2}-\left(d_{1}-1 / 2\right)^{2}+1 / 4\right)^{2}=0 .
\end{aligned}
$$

Hence one line segment of the boundary of $S$ is mapped to the line segment $-1 / 4 \leq x \leq$ $1 / 4$ of the boundary $H$ (see Figure 2). Similarly, from the other equations, we see the boundary behaviour by the bilateration mapping.

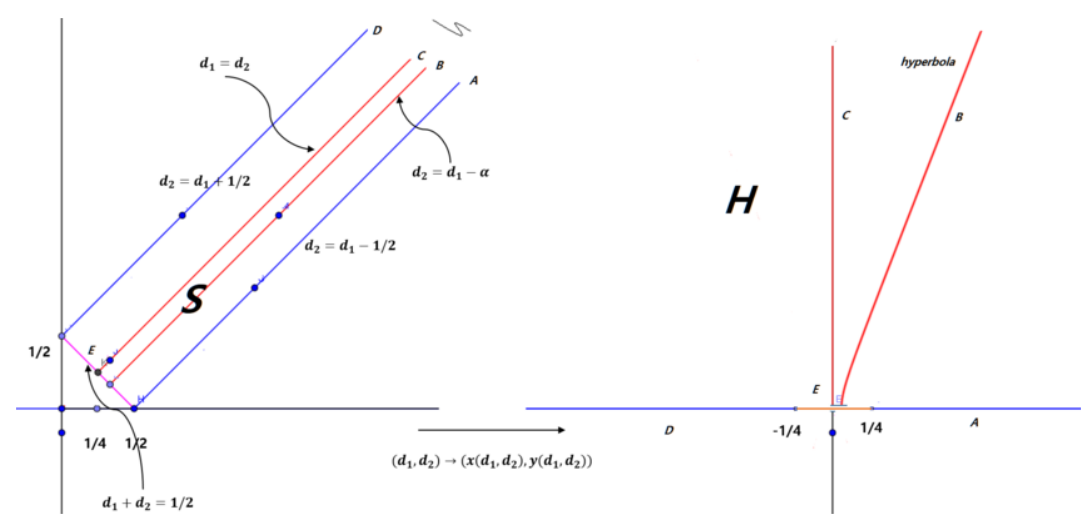

Figure 2. Bilateration Mapping 
Note. The lines $d_{2}=d_{1}-\alpha(0 \leq \alpha \leq 1 / 2)$ in $S$ are mapped to the hyperbolas (see the red lines in Figure 2), since $d_{1}-d_{2}=\alpha$ (constant) defines one piece in the right half plane of hyperbola (note that it define a hyperbola if the difference of the distance $\overline{P A_{1}}$, $\overline{P A_{2}}$ two known nodes $A_{1}, A_{2}$ is constant).

Theorem 2.2. The bilateration mapping $\left(d_{1}, d_{2}\right) \mapsto(x, \pm y)$ of Theorem 2.1 exactly gives the localization mapping from $S \times\{+1,-1\}$ to $\mathbb{R}^{2}$.

Next section ( $\S 3$ ), we give a practical way how to choose \pm 1 (upper half plane, or lower half plane).

Proof. By (3) and (4), the function $\left(d_{1}, d_{2}\right) \mapsto\left(x\left(d_{1}, d_{2}\right),+y\left(d_{1}, d_{2}\right)\right)$ maps $S$ to the upper half plane (see the proof of Theorem 2.1 and Figure 2). Also, $\left(d_{1}, d_{2}\right) \mapsto$ $\left(x\left(d_{1}, d_{2}\right),-y\left(d_{1}, d_{2}\right)\right)$ maps $S$ to the lower half plane, since the lower half plane is the mirror reflection of the upper half plane with respect to the $x$-axis, and the $y$-component has negative values in lower half plane,

\subsection{Change of Variables: New Expression}

In trilateration method (see Figure 1b), it is known [8,9] that the coordinates of the unknown node is represented by the following hyperbolic equation

$$
x=\left(d_{1}^{2}-d_{2}^{2}+1\right) / 2, \quad y=\left(d_{1}^{2}-d_{3}^{2}+1\right) / 2 .
$$

Similarly, up to change of variables, we have also hyperbolic equations in bilateration.

Theorem 2.3. By almost linear change of variables, that is, squared mapping $t \mapsto t^{2}$ and linear transformation (including rotation translation), we have

$$
\begin{aligned}
& x\left(d_{1}, d_{2}\right)=\left(d_{1}+d_{2}\right)\left(d_{1}-d_{2}\right) \rightsquigarrow \sqrt{\left(u_{1}^{2}-u_{2}^{2}\right) / 2} \\
& y\left(d_{1}, d_{2}\right)=\left[d_{1}^{2}-\left(d_{1}^{2}-d_{2}^{2}+1 / 4\right)^{2}\right]^{1 / 2} \rightsquigarrow \sqrt{\left(v_{1}^{2}-v_{2}^{2}\right) / 2}
\end{aligned}
$$

Proof. Let $s=d_{1}+d_{2}$, and $t=d_{1}-d_{2}$, and $\tilde{u}_{1}=s^{2}, \tilde{u}_{2}=t^{2}$. Then, rotating the curve by $(-\pi / 4)$ around the origin,

$$
\begin{aligned}
x^{2} & =\left(d_{1}^{2}-d_{2}^{2}\right)=\left(d_{1}+d_{2}\right)^{2}\left(d_{1}-d_{2}\right)^{2} \\
& =s^{2} t^{2}=\tilde{u}_{1} \tilde{u}_{2} \rightsquigarrow\left(u_{1}^{2}-u_{2}^{2}\right) / 2 .
\end{aligned}
$$

Next, consider the equation of $y$-component. By factoring the term $y^{2}=d_{1}^{2}-\left(d_{1}^{2}-\right.$ $\left.d_{2}^{2}+1 / 4\right)^{2}$, we have

$$
\begin{aligned}
y^{2} & =\left(d_{1}-\left(d_{1}^{2}-d_{2}^{2}+1 / 4\right)\right)\left(d_{1}+\left(d_{1}^{2}-d_{2}^{2}+1 / 4\right)\right) \\
& =-\left(\left(d_{1}-1 / 2\right)^{2}-d_{2}^{2}\right)\left(\left(d_{1}+1 / 2\right)^{2}-d_{2}^{2}\right) \\
& =-\left(d_{1}-1 / 2-d_{2}\right)\left(d_{1}-1 / 2+d_{2}\right)\left(d_{1}+1 / 2-d_{2}\right)\left(d_{1}+1 / 2+d_{2}\right) \\
& =-\left[\left(d_{1}+d_{2}\right)^{2}-(1 / 2)^{2}\right]\left[\left(d_{1}-d_{2}\right)^{2}-(1 / 2)^{2}\right]
\end{aligned}
$$


Letting $s=d_{1}+d_{2}$, and $t=d_{1}-d_{2}$, and again $\tilde{v}_{1}=s^{2}-1 / 4$, and $\tilde{v}_{2}=t^{2}-1 / 4$,

$$
y^{2}=-\left(s^{2}-1 / 4\right)\left(t^{2}-1 / 4\right)=-\left(\tilde{v}_{1} \tilde{v}_{2}\right) \rightsquigarrow\left(v_{1}^{2}-v_{2}^{2}\right) / 2
$$

by $(\pi / 4)$-rotation.

\section{Application of Bilateration to the human-following robot}

Let $C$ be a curve in the plane. Now, assume the moving frame on $\mathbb{R}^{2}$ along the curve $C$, which just means the Cartesian coordinate system ${ }^{4}$ of the Euclidean plane $\mathbb{R}^{2}$ that moves with the observer along $C$ (see Figure 3a).

To develop human-following robot based on the position of leading human, it is necessary to consider only the relative position of human with respect to the robot. Equivalently, from the following robot's view point, it is better to consider the moving coordinate system mounted on the robot, since the robot follows it as human is moving along $C$. In this case, by rotating the robot fixing the origin $O$, we can determine the right solution from the two candidates coordinates in Theorem $\mathbf{2 . 2}$ of the human, which are calculated by the proposed bilateration. It is known [10] that the human-following robot based on the position has fundamental advantages compared to the vision-based one [11].

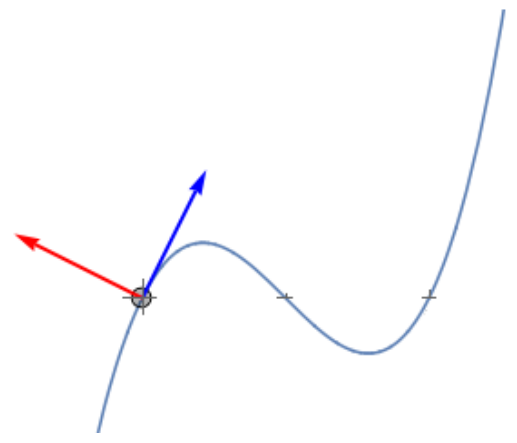

(a) Moving frame in $\mathbb{R}^{2}$

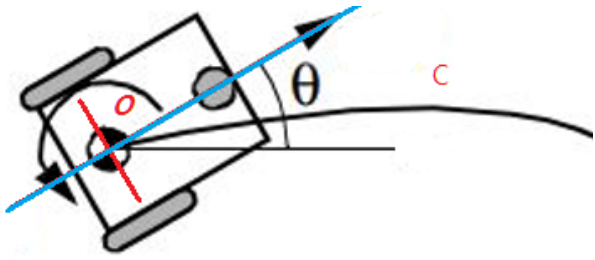

(b) Moving frame mounted on the robot

Figure 3. Bilateration based on moving frame for the human-following robot

\section{Conclusion}

In this paper we have studied the mapping properties of the bilateration method and proposed the bilateration for the concept model of the human-following robot, since the relative position of the leading human with respect to the robot is easily obtained and the bilateration needs only two sensor nodes mounted on the robot, which results in very simple robot's architecture.

With regard to the mapping property of the bilateration, first, we showed how two measured distances maps into the position of the unknown node (Theorem 2.1 and The-

\footnotetext{
${ }^{4}$ orthonormal frame
} 
orem 2.2 ). Second, we proved that the coordinates functions of the unknown node by the bilateration essentially have the same hyperbolic equation as the trilateration after changing almost linear change of variables (Theorem 2.3).

In general, though the bilateration has one more ambiguous coordinates for the unknown node, we can overcome this disadvantage by mounting the sensors on the mobile robot and moving (or rotating) the robot or sensors.

The proposed bilateration has more advantages compared to other localization techniques like multilateration, since we have only two measurement errors in bilateration, from which the number of the required nodes, computation time, and the response time can be reduced and hence better positioning accuracy is expected.

\section{References}

[1] Zafari F, Gkelias A, Leung KK. A Survey of Indoor Localization System and Technologies. IEEE Comm. Surveys \& Tutorials. 2019; 21(3): 2568-2509.

[2] Perera, C.; Liu, C. H.; Jayawardena, S. (December 2015). The Emerging Internet of Things Marketplace From an Industrial Perspective: A Survey. IEEE Transactions on Emerging Topics in Computing. 2015 Dec; 3(4): 585-598.

[3] Want, R; Schilit, BN.; Jenson, S. Enabling the Internet of Things. Computer. 2015 Dec; 48: 28-35.

[4] Safavi S, Khan UA, Kar S, Moura JMF. Distributed Localization: A Linear Theory. Proc of IEEE. 2018 July; 106(7): 1204-1223

[5] Cota-Ruiz J, Rosiles JG, Sifuentes E, Rivas-Perea P. A Low-Complexity Geometric Bilateration Method for Localization in Wireless Sensor Networks and Its Comparison with Least-Squares Methods, Sensors. 2012 Dec; 839-862

[6] Li X, Hua B, Shang Y, Xiong Y. A robust localization algorithm in wireless sensor networks, Front. Comput. Sci. China 2008; 2(4): 438-450

[7] Tiwari S, Jain VK. Heron-Bilateration based Location Estimation Technique for Indoor WLAN, In: Proceeding of the 31st International Conference on Information Networking (ICOIN 2017); 2017 Jan 11-13; Da Nang, Vietnam: IEEE PDF eXpress; p. 13-17

[8] Lee SC, Rizal S, Ahn H. Anchor Placement Design for a Decoupled Simple Trilateration Algorithm. Journal of Nonlinear and Convex Analysis. 2019; 20(7); 1327-1339

[9] Lee SC, Ahn H., A New Mobile Real-Time Localization System for a Following Robot Application, Journal of Nonlinear and Convex Analysis. 2020; 21(7); 1477-1485

[10] Feng T, Yu Y, Wu L, Bai Y, Xiao Z, Lu Z. A Human-Tracking Robot Using Ultra Wideband Technology. IEEE Access. 2018; 6: 42541-42550

[11] Gupta M, Kumar S, Behera L, Subramanian VK. A Novel Vision-Based Tracking Algorithm for a Human-Following Mobile Robot. IEEE Trans on Systems. 2017 July; 47(7): 1415-1427 